LBL-33190

UC -410

LSGN-105

\title{
THE PHASE SERVO TUNER CONTROL SYSTEM OF THE ALS SOOMHZ CAVITY
}

\author{
C.C. LO and BRIAN TAYLOR \\ ACCELERATOR and FUSION RESEARCH DIVISION \\ Lawrence Berkeley Laboratory \\ University of California \\ Berkeley, CA 94720
}

MAY 1993

\section{MASTER}

This work was supported by the Director, Office of Energy Research, Office of Basic Energy Sciences, Materials Sciences Division, of the U.S. Department of Energy under Contract No. DE-AC03-76SF00098. 


\title{
The Phase Servo Tuner Control System of The ALS 500MHz Cavity*
}

\author{
C.C. Lo and Brian Taylor \\ Lawrence Berkeley Laboratory, University of California \\ 1 Cyclotron Road, Berkeley, CA 94720 USA
}

\section{Abstract}

Three $500 \mathrm{MHz}$ cavities are used in the Booster and Storage Ring of the Advanced Light Source (ALS). Due to different varying parameters, a control system is required to keep the cavities in tune during operation. The tuning of the $500 \mathrm{MHz}$ cavity is achieved by detecting the phase error between the drive signal and the cavity probe signal. The error signal is amplified and used to drive a stepping motor which in turn moves a metallic cylinder in or out of the cavity to achieve tuning.

\section{INTRODUCTION}

There is one cavity[1] in the Booster and two in the Storage Ring. The tuning of each of these cavities is controlled by a Phase Servo Tuner Control Module. The tuner, a metallic cylinder, is driven by a five phase stepping motor, which in turn is driven by a driver. The driver accepts a bipolar input signal derived from a phase detector in the Phase Servo Tuner Control Module. The amplitude and the polarity of the signal determines the speed and the direction of rotation of the motor. The resolution of the stepping motor is 0.5 degree of rotation per step.

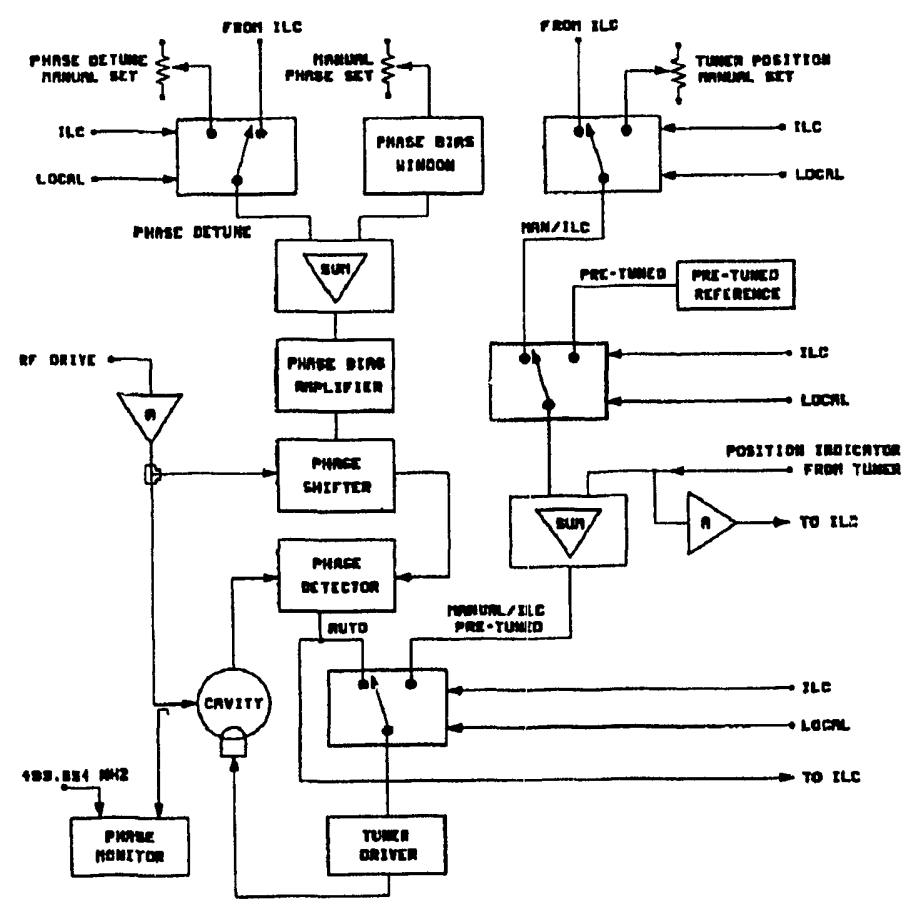

XBL 934-536

Figure 1. Block diagram of the control module.

*This work was supported by the Director, Office of Energy Research, Office of Basic Energy Sciences, Materials Sciences Division, of the U.S. Department of Energy under Contract No. DEAC03-76SF00098.
Figure 1 shows the block diagram of the control module. Most controls have been implemented with the capability of operating under local controls or remotely via the Intelligent Local Controller (ILC)[2] and the computer in the main control room. With the exception of some discrete RF components all electronic circuits are built on a printed circuit board with a ground plane. Figure 2 is a photograph of the component layout of the module which is a standard 19" rack mount unit. Figure 3 is a photograph of the front panel of the module which shows practically all of the functions the system is capable of performing.

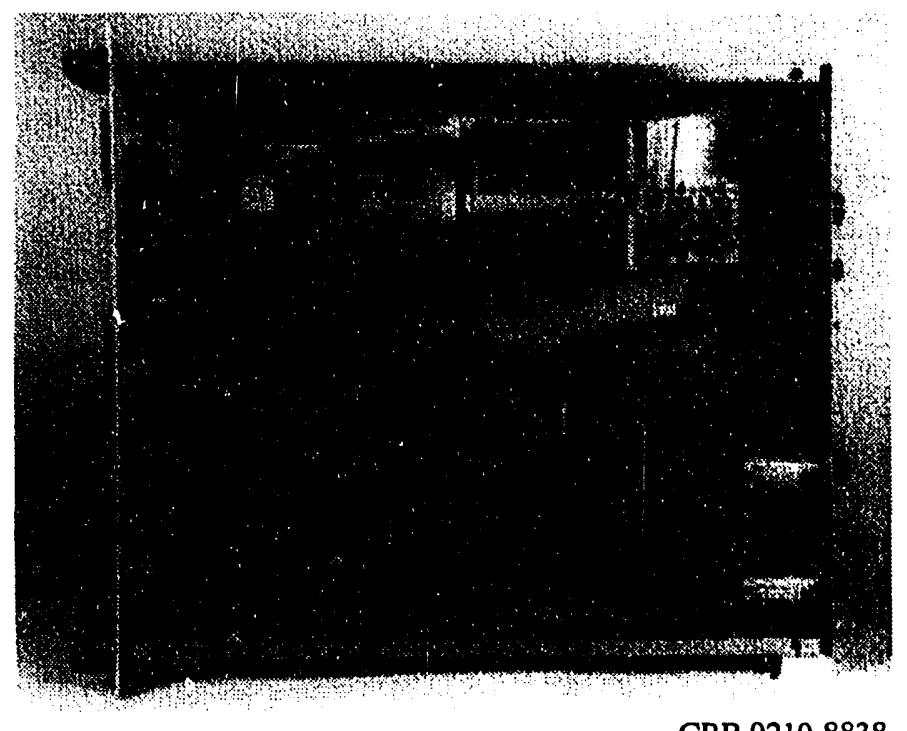

Figure 2. Component layout of the Module.

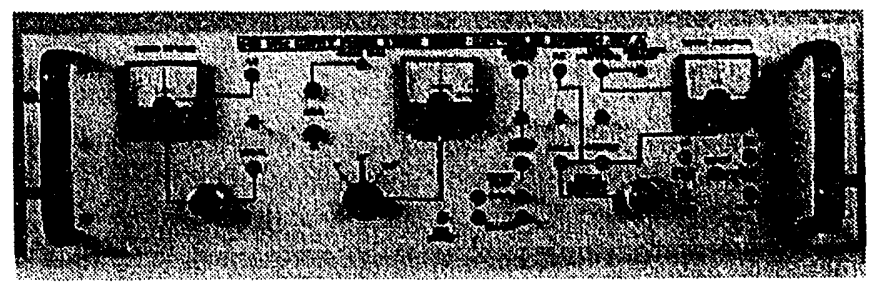

CBB 9210-8852

Figure 3. Front view of the module.

\section{TUNER POSITION CONTROLS}

The tuner position can be set either locally or remotely by the ILC. The total travel of the tuner is approximately $10 \mathrm{~cm}$. A pot attached to the tuner provides the feedback signal for the control module. The two electronic limit switches of the tuner are set by two adjustments in the control module which shuts 
down the drive to the motor below $20 \%$ and above $80 \%$ of the travel. Should one of these switches be activated a drive signal for the other direction is able to put the tuner back in the normal operating range. The two mechanical limit switches in the tuner provide additional insurance to protect the tuner from ramming the end points of the travel. If for any reason one of these mechanical switches is activated the tuner can be restored to the operating range by two push button switches together with a logic circuit.

A pretuned position setting has also been implemented to bring the tuner back to the tuned position at any time by activating the pretuned switch. This is particularly useful in cases where the tuner is at some arbitrary position during an interrupted operation.

\section{TUNING THE CAVITY}

A reference signal and a RF sample signal from the cavity are required to produce a tuner correction signal with a phase detector. A double balanced mixer is used as the phase detector. In the reference signal path, a mechanical and an electronic phase shifter together with an attenuator are put in series for phase and amplitude offset and adjustment purposes. The RF signal for the signal port is derived from the cavity monitor. Again, proper attenuators are used to bring the signal level down to a range of $+3 \mathrm{dBm}$ to $+20 \mathrm{dBm}$ with the nominal level center around $+13 \mathrm{dBm}$ at the signal input ports of the phase detector. A transfer function of the phase error and the DC output voltage from the phase detector is given in figure 4.

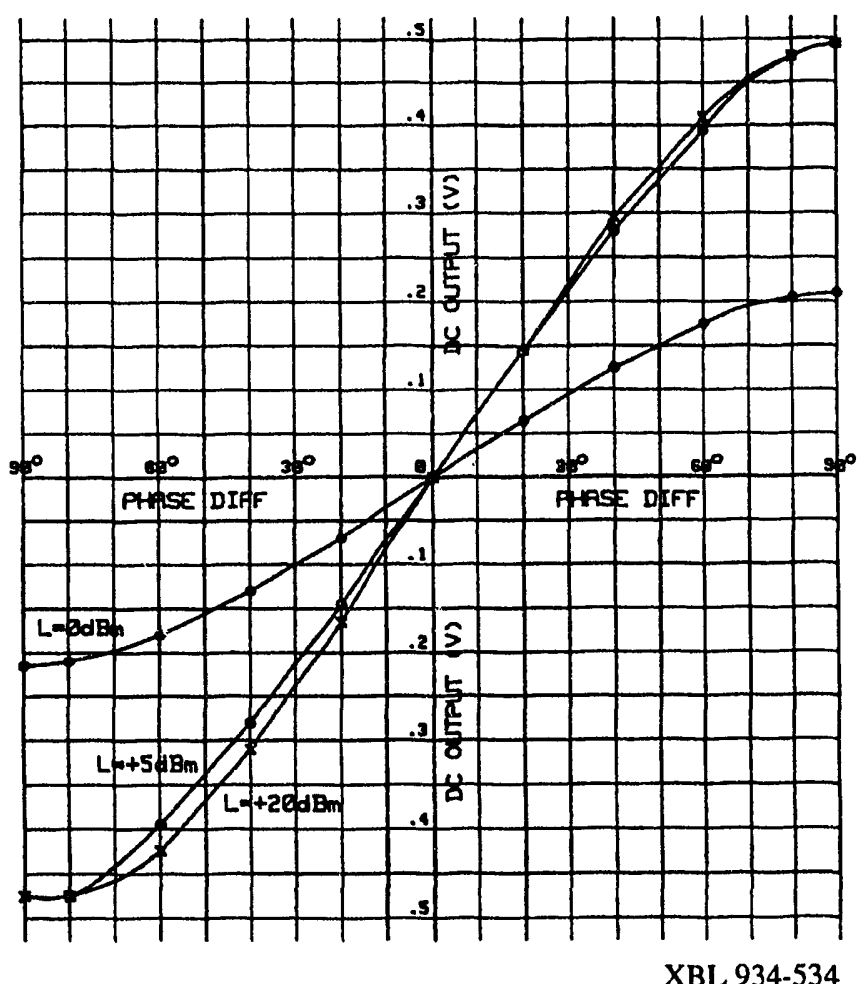

Figure 4. Transfer function of the phase detector.
The signal input port of the phase detector is used as the reference signal port and the local oscillator input port is used as the RF signal port. The reference signal level for this measurement was $+5 \mathrm{dBm}$. The resolution of the phase detector is approximately $7 \mathrm{mV}$ per degree. With the proper amplification of this error signal matching the resolution of the servo driver of the tuner, tuning resolution of approximately 0.1 degree is achieved. The tuning error of the tuning systern at the nominal operating power level is of the same order as the tuning resolution. Therefore, the over all worst case phase error should still be better than 0.25 degree. The tuner servo motor tuning rate is $114 \mathrm{KHz}$ per second, therefore an $11.4 \mathrm{KHz}$ detuning would require 0.1 second to get the cavity back in tune. The dynamic operating range without adjusting any attenuation in both signal paths is approximately $23 \mathrm{~dB}$, whereas the phase detection range is $+/-90$ degrees. Both parameters have been shown to be quite adequate in day to day operation.

\section{PHASE DETUNING OF CAVITY}

An electronic phase shifter is used to adjust the phase of the reference signal in the case of closed loop operation either to fine tune the phase detector or to detune the cavity by offsetting the tuner to resonate the cavity at a lower frequency by approximately $10 \mathrm{KHz}$. The detuning is used mainly to counteract instability due to beam loading. Figure 5 shows the typical detuned phase angle and detuned frequency as functions of the detune bias voltage of the servo control modules. The detuned phase angle transfer function was measured by observing the phase angle between the waveguide signal and the cavity signal while changing the detune bias.

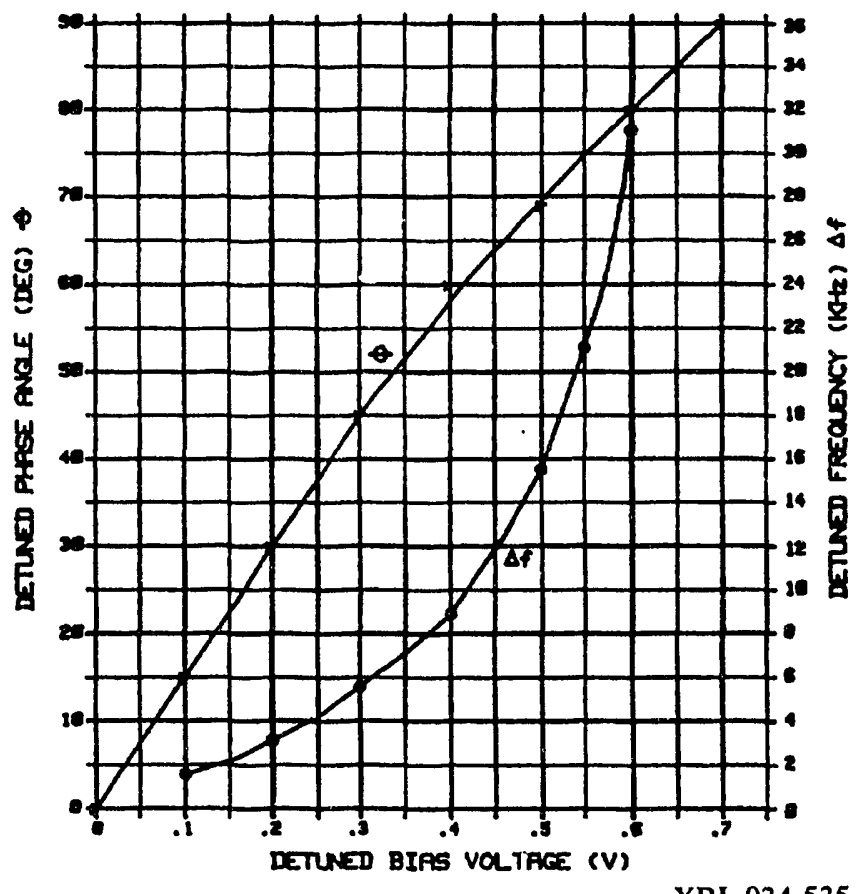

Figure 5. Transfer functions of the detuned phase angle and detuned frequency. 
The detuned frequency is calculated by the following expression:

$$
\Delta f=\frac{\mathrm{a}}{\mathrm{Q}} \mathrm{X} \text { fo }
$$

where $\Delta f=$ detuned frequency

$a=a$ constant obtained from the Universal resonance curve[3]

$\mathrm{Q}=\mathrm{Q}$ of the cavity

fo $=$ resonant frequency

The two cavities in the storage ring have been operating with a detune bias voltage of approximately $0.4 \mathrm{~V}$. The $Q$ of the cavity is approximately 45,000 .

The best operating range of the phase shifter is used by selecting the proper bias voltage range. The local phase bias is set at the mid range of the phase shifter to provide enough room on both sides for the phase detune to work properly. However, under normal operation, the phase angle only needs to be detuned to the lower frequency side. Because the phase shifters used in the servo control modules have different transfer functions, variations in detune voltages from different modules should be expected.

\section{CONCLUSION}

One Phase Servo Tuner Controller Module has been operating in the cavity test stand for over two years, one in the Booster Ring for over a year, and two have been in operation in the Storage Ring for a couple of months. All four units have been performing well. Network analyzers with Polar displays have been used to monitor the operation of the storage ring cavities. At the time of the writing of this report, the capability of computer tuning of the cavities has not been implemented. Operating experience will tell whether this procedure will be necessary.

\section{ACKNOWLEDGMENTS}

This work was funded by the director, Office of Energy Research, Office of Basic Energy Sciences, Material Science Division of the U.S. Department of Energy under Contract No. DE-AC03-76SF00098 with Lawrence Berkeley Laboratory. Reference to a company or a product name does not imply approval or recommendation of the product by the University of California or the U.S. Department of Energy to the exclusion of others that may be suitable.

\section{REFERENCES}

[1] C.C. Lo and Brian Taylor, "Computer-Aided Studies of the ALS $500 \mathrm{MHz}$ Storage Ring Cavity", Proceedings of 1989 IEEE PAC, Vol. 1, pp. 121-123, LBL-25976.

[2] S. Magyary, et al, "Advanced Light Source Control System", Proceedings of 1989 IEEE PAC, Vol. 1, pp.7478, LBL 28412.

[3] F.E. Terman, Electronic and Radio Engineering, McGraw Hill Book Company, Inc. 1955. 

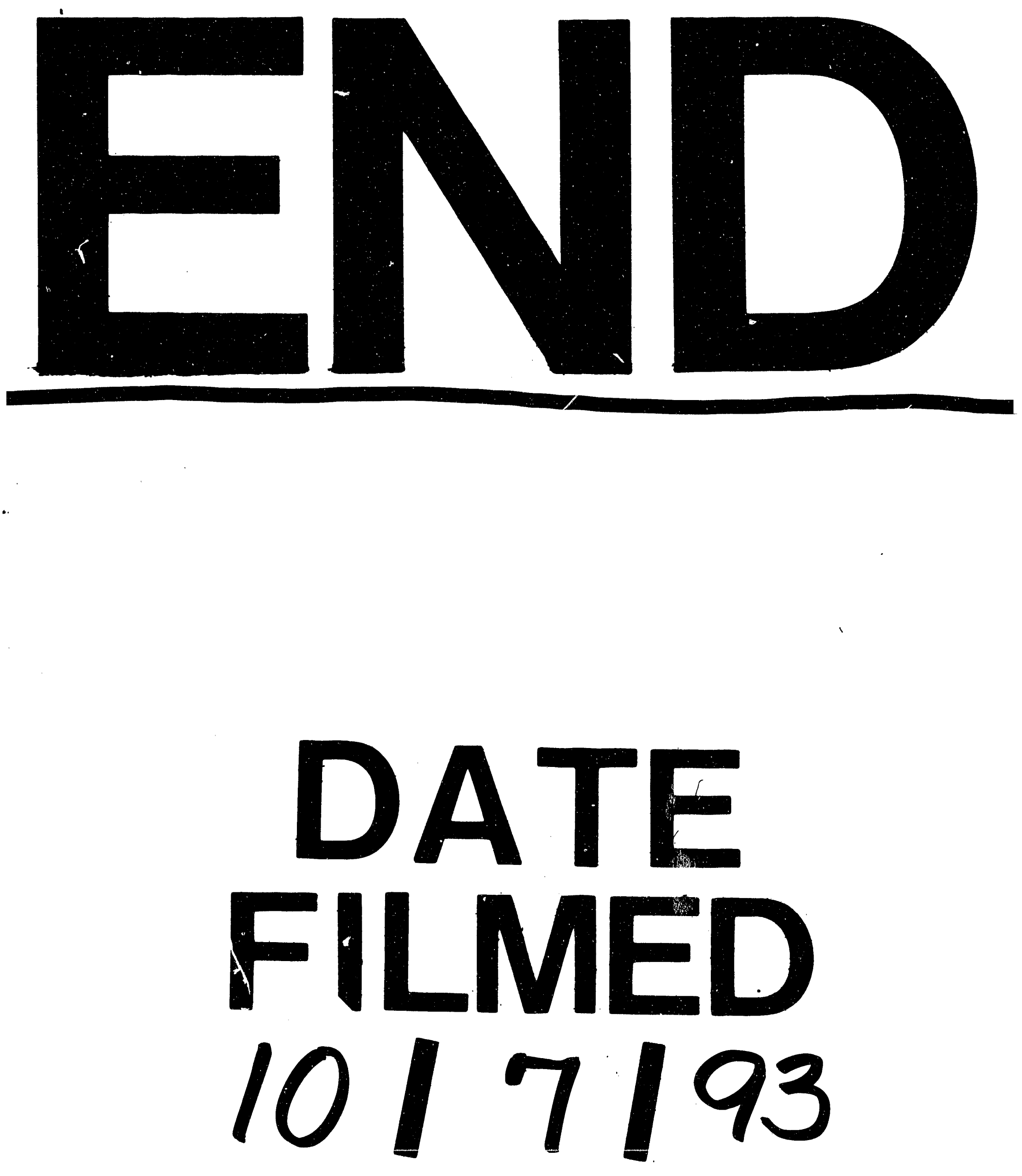
